\title{
Statistical Methods in the Evaluation of Cardio-Respiratory Parameters in Young Childhood Cancer Survivors and Healthy Peers
}

\author{
Magdalena Topczewska ${ }^{1}$, Małgorzata Sawicka-Żukowska ${ }^{2}$, \\ Joanna Zapolska ${ }^{3}$, Lucyna Ostrowska ${ }^{3}$, Maryna Krawczuk-Rybak ${ }^{2}$ \\ 1 Faculty of Computer Science, Bialystok University of Technology, Poland \\ 2 Department of Pediatric Oncology and Haematology, Medical University of Bialystok, \\ Poland \\ 3 Department of Dietetics and Clinical Nutrition, Medical University of Bialystok, Poland
}

\begin{abstract}
This study concerns the problem of late complications of antineoplastic therapy. Reduced parameters of the cardiorespiratory system in childhood may have a tremendous impact on health. In order to assess the selected parameters, to evaluate physical endurance, and compare the results with those obtained for healthy children, a test was carried out on a treadmill, until $80 \%$ of maximum pulse rate was reached. To compare the differences between the treatment group and the control group, three approaches were used. The first one was the classical statistical inference, the second consisted in forming a multidimensional normal model and also involved modelling of the correlation between variables. The unstructured type of the working correlation matrix was chosen to obtain the results and correct standard errors. In the last approach, logistic regression was used to model the relationship between binary outcome and covariates, and to differentiate between the groups of patients on the basis of their cardiovascular parameters.
\end{abstract}

\section{Introduction}

Among the late complications of antineoplastic therapy, overweight and obesity are the main ones. The occurrence of these two types of complications in the group of children treated for cancer can be influenced by the use of chemotherapy and steroid therapy. Furthermore, immobilization and the lack of activity during treatment as well as prolonged neutropenia may constitute another reason. Completion of therapy should lead to the temporarily decreased levels of physical activity returning to their normal levels. However, this does not occur for a large fraction of cancer survivors. This 
may carry the risk of occurrence of similar problems, with consequences in adulthood.

With the increase in the number of obesity cases among children, the number of studies on this issue is also growing. The consequences and risks of childhood and adolescent overweight and obesity can be short-, mid-, or long-term. The psychological and social effects are the most widespread (Dietz, 1998) and can lead, for instance, to anxiety and depression (Morrison et al., 2015), or bullying and stigma (Beck, 2016). The consequences can also be associated with various serious health problems, including high blood pressure and high cholesterol levels (Cote et al., 2013; Lloyd et al., 2012); pulmonary (asthma, sleep apnea - Mohanan et al., 2014; Narang \& Mathew, 2012); and skeletal (Pollock, 2015); along with increased risk of developing diabetes (Bacha \& Gidding, 2016), and heart disease (Cote et al., 2013; McCrindle, 2015). Lauer R. M. and Clarke W. R. (1989) noticed that obese boys and obese girls (BMI>90th percentile) are 9-10-fold more likely to develop high blood pressure as young adults than non-obese children. Childhood obesity is also associated with a 50\% higher risk of urothelial or colorectal cancers (Leiba et al., 2012).

Early prevention of overweight and obesity becomes one of the main objectives following anticancer therapy, particularly in relation to young survivors. Keeping a healthy weight, normal fat distribution and appropriate exercise parameters reduce the risk of cancer.

In order to check the overall condition of the body, efficiency and endurance parameters can be used. The aim of the study was to assess selected cardiorespiratory parameters after the completion of antitumor therapy and to compare them with the same parameters in healthy children.

\section{Data}

The dataset was obtained from the Department of Pediatric Oncology and Haematology, Medical University of Bialystok, and contained the values of variables for 72 children belonging to the group of those treated for cancer (treatment) or the control group. The control group consisted of healthy children who were siblings of the treated patients or children hospitalized for reasons other than cancer.

The set of variables consisted of two subsets. The first subset of attributes was formed by performing eleven general measurements such as: group indication, gender, diagnosis, bone marrow transplant, steroid use, irradiation of the central nervous system, age during research, age at diag- 
nosis, age at completion of anticancer treatment, weight SDS and BMI SDS. The last two variables were presented as standard deviation scores (SDS). Topczewska et al. (2014) mentions that of the 72 children, $48.61 \%$ (35 children) were in the treatment group and $51.39 \%$ (37 children) in the control group; males constituted $47.22 \%$ (34), females $52.78 \%$ (38) of the whole group. There were 18 females and 17 males in the treatment group, and 20 females and 17 males in the control group. Out of the 35 patients, $85.71 \%$ (30) did not undergo bone marrow transplant while 5 patients did; $65.71 \%$ (23) of the patients took steroids during treatment while $34.29 \%$ (12) did not; $34.29 \%$ (12) underwent irradiation of the central nervous system while $65.71 \%$ (23) did not.

Another subset of attributes described the parameters of the cardiovascular system. The study was carried out on a treadmill, i.e. the child was walking wearing a mask until $80 \%$ of maximum pulse rate was reached. Two tests were performed simultaneously: an exercise (stress) test and a pulmonary test. The aim of the exercise test was to evaluate exercise tolerance, which meant obtaining a response to the effort of the respiratory, cardiac, and musculoskeletal systems: metabolic gas exchange and gas exchange in the lungs (oxygen consumption, carbon dioxide production), ventilation (minute ventilation), and cardiovascular response, in addition to monitoring ECG and blood pressure. Among the recorded parameters, four were chosen for further analysis: metabolic equivalent - rest (idle) oxygen consumption, maximal oxygen uptake $[\mathrm{ml} / \mathrm{min}]$, maximal heart rate $[\mathrm{sk} / \mathrm{min}]$, maximal VO2 at heart rate $[\mathrm{ml} / \mathrm{sk}]$.

According to the definition, one metabolic equivalent of task ( $1 \mathrm{MET})$ is the amount of oxygen consumed while sitting at rest and is equal to $3.5 \mathrm{ml} \mathrm{O}_{2}$ per kg body weight min (Jetté et al., 1990). It expresses the energy cost of physical activity. Maximal oxygen uptake is the maximum amount of oxygen the body can take during an exercise requiring near-maximal or maximal exertion. Heart rate is the rate at which the heart beats per minute, while maximal heart rate is the highest number of beats per minute the heart can pump under maximum effort. A high maximal oxygen uptake value and a low resting heart rate are both associated with good cardiovascular fitness.

The first and the fourth attributes were continuous, while the second and the third ones were discrete variables with various ranges. A small inconvenience connected with these variables is the following: the norm for each of the variables changes in time, thus it varies for different ages and different genders. For this reason, deviations between the original values and the corresponding norms were calculated. These new values were then used in the analysis. 


\section{Methods}

For a comparison between the groups, an assumption about normal distribution was checked with the use of the Shapiro-Wilk test. If the assumption was fulfilled, the assumption about variance equality was checked. The $t$ test was performed in order to make a comparison between the two groups. If unequal variances were present, Welch approximation was used. In the case of non-normal distributions, the Kruskal-Wallis test was used.

For normally distributed data, the mean values with standard deviation are presented in the report, while for non-normally distributed data, the medians and ranges are shown. The differences in proportions were checked using the Fisher exact test. Statistical significance at the level of 0.05 was taken. To model the relationship between binary output and independent variables, logistic regression was chosen. Stata IC/11.0 was used to perform statistical calculations. To build the multivariate normal model, the SAS System was used. To assess the classification accuracy as well as sensitivity and specificity with the use of k-folds, a cross-validation technique, i.e. Weka System, was used. The number of folds was fixed at 10 .

\section{Results}

Hypothesis testing. The primary approach to comparison of groups of objects is statistical inference and hypothesis testing. The calculations refer to comparisons between two groups of patients and concern the mean values of deviations from the norm, constituting an attempt to assess whether the four different parameters vary between the two groups. Firstly, the grouping variable belongs either to the treatment group or to the control group. Then, it also belongs to one of the gender subgroups and may belong to a group defined by the occurrence of a special procedure, such as bone marrow transplant, during treatment.

At a significance level of 0.05 , it was checked and confirmed that all four variables are normally distributed in the groups, the gender subgroups, and all the other subgroups considered in this paper; hence, the ranges of values are presented for informative purposes only, mainly due to the fact that deviations between the original values and the corresponding norms were analyzed.

The results of the comparison between the treatment group and the control group are presented in Table 1 and in Figure 1. Among the selected cardiorespiratory parameters, statistically significant differences can be ob- 
Table 1. Comparisons between the treatment group and the control group for cardiovascular parameters

\begin{tabular}{|l|r|r|r|c|r|r|r|c|c|}
\cline { 2 - 9 } \multicolumn{1}{c|}{} & \multicolumn{4}{c|}{ Treatment group } & \multicolumn{4}{c|}{ Control group } & \multirow{2}{*}{$p$} \\
\cline { 2 - 9 } & $n$ & \multicolumn{1}{c|}{$\bar{x}$} & \multicolumn{1}{c|}{$s d$} & range & $n$ & \multicolumn{1}{c|}{$\bar{x}$} & \multicolumn{1}{c|}{$s d$} & range & \\
\hline Metabolic equivalent & 35 & -3.5 & 2.0 & $-8.7 ;-0.3$ & 37 & -2.5 & 1.7 & $-7.5 ; 1.1$ & 0.031 \\
Maximal oxygen uptake & 35 & -672.0 & 341.2 & $-1387 ;-99$ & 37 & -521.5 & 414.8 & $-1508 ; 385$ & 0.098 \\
Maximal heart rate & 35 & -24.4 & 5.8 & $-40 ;-10$ & 37 & -27.9 & 5.8 & $-43 ;-14$ & 0.009 \\
Max. $\mathrm{VO}_{2}$ at a heart rate & 35 & -2.3 & 2.1 & $-6.4 ; 2.0$ & 37 & -1.2 & 2.3 & $-6.6 ; 4.4$ & 0.039 \\
\hline
\end{tabular}

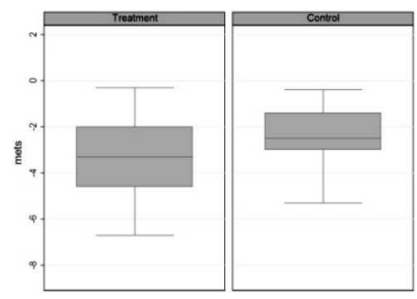

a) Metabolic equivalent

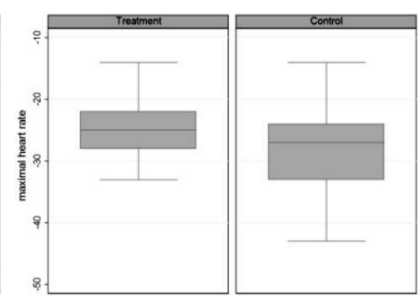

b) Maximal heart rate

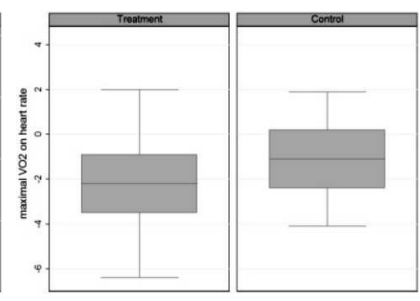

c) Maximal VO2 at a heart rate

Figure 1. Box plots for three parameters for the treatment group and the control group

served for three variables: metabolic equivalent $(-3.5 \pm 2.0$ for the treatment group versus $-2.5 \pm 1.7$ for the control group; $p=0.031)$; maximal heart rate $(-24.4 \pm 5.8$ for the treatment group versus $-27.9 \pm 5.8$ for the control group; $p=0.009)$ and maximal $\mathrm{VO}_{2}$ at a heart rate $(-2.3 \pm 2.1$ for the treatment group versus $-1.2 \pm 2.3$ for the control group; $p=0.039)$. The absolute values of average deviations from the norm are given for these two attributes and are larger for the treatment group. The values are negative, which indicates a smaller metabolic equivalent and a smaller maximal $\mathrm{VO}_{2}$ at a heart rate for cancer survivors compared to healthy children.

Differences between average deviations for the four cardio-respiratory parameters were also assessed for the gender subgroups (Table 2 and 3). Statistically significant differences in the results between the treated and the healthy children for all the four attributes were only observed for the female subgroup (Table 3, Figure 2), while in the case of the male subgroup, the differences in the mean values were insignificant (Table 2). This may lead to the conclusion that regaining physical fitness at the healthy children level described by the chosen parameters, can only be observed for males, whereas many females remain at the limited level of physical activity. 
Table 2. Comparison of cardiovascular parameters between the treatment group and the control group for the male subgroup

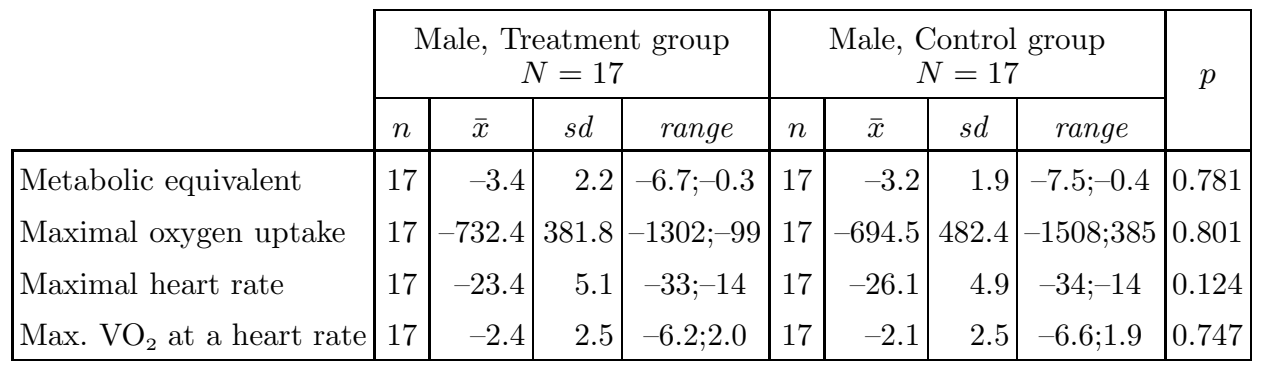

Table 3. Comparisons of cardiovascular parameters between the treatment group and the control group for the female subgroup

\begin{tabular}{|c|c|c|c|c|c|c|c|c|c|}
\hline & \multicolumn{4}{|c|}{$\begin{array}{l}\text { Female, Treatment group } \\
\qquad N=18\end{array}$} & \multicolumn{4}{|c|}{$\begin{array}{c}\text { Female, Control group } \\
\qquad N=20\end{array}$} & \multirow[t]{2}{*}{$p$} \\
\hline & $n$ & $\bar{x}$ & $s d$ & range & $n$ & $\bar{x}$ & $s d$ & range & \\
\hline Metabolic equivalent & 18 & -3.6 & 1.9 & $-8.7 ;-1.1$ & 20 & -1.9 & 1.4 & $-4.7 ; 1.1$ & 0.004 \\
\hline Maximal oxygen uptake & 18 & -614.9 & 297.6 & $-1387 ;-268$ & 20 & -374.5 & 283.7 & $-883 ; 372$ & 0.015 \\
\hline Maximal heart rate & 18 & -25.3 & 6.4 & $-40 ;-10$ & 20 & -29.6 & 6.1 & $-43 ;-21$ & 0.041 \\
\hline Max. $\mathrm{VO}_{2}$ at a heart rate & 18 & -2.3 & 1.7 & $-6.4 ; 0.1$ & 20 & -0.5 & 1.9 & $-4.1 ; 4.4$ & 0.005 \\
\hline
\end{tabular}

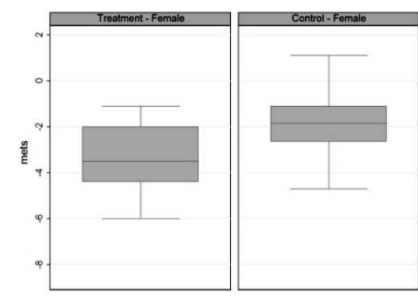

a) Metabolic equivalent

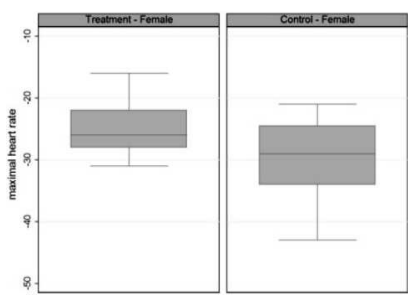

c) Maximal heart rate

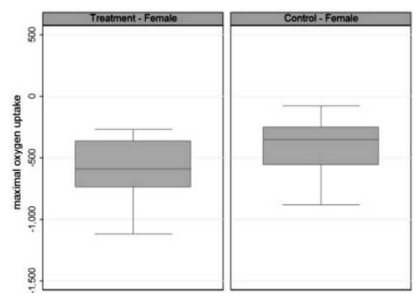

b) Maximal oxygen uptake

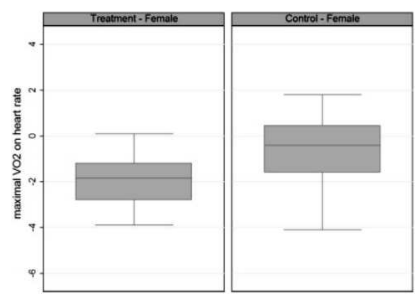

d) Maximal VO2 at a heart rate

Figure 2. Box plots for four cardiovascular parameters for the treatment and the control group for the female subgroup 
Considering only the treatment group, the differences between the additional subgroups were calculated to check if the time since treatment completion, the occurrence of bone marrow transplant, taking steroids, or central nervous system irradiation have an impact on the results. The first analysis referred to the time since treatment completion, with 5 years as the discriminatory value. The results are presented in Table 4. In Table 5, calculations for the presence of bone marrow transplant are shown, while in Table 6 variables are compared for the steroids group; finally, in Table 7 the variables are compared for the central system irradiation group.

Table 4. Comparisons between two groups (time since treatment completion, from 1 to 5 years versus over 5 years) for 4 cardiovascular parameters

\begin{tabular}{|c|c|c|c|c|c|c|c|c|c|}
\hline & \multicolumn{4}{|c|}{$\begin{array}{l}\text { Treatment group } \\
\text { Time to treatment finish } \\
\text { from } 1 \text { till } 5 \text { years } \\
N=12\end{array}$} & \multicolumn{4}{|c|}{$\begin{array}{l}\text { Treatment group } \\
\text { Time to treatment finish } \\
\text { above } 5 \text { years } \\
N=13\end{array}$} & \multirow[t]{2}{*}{$p$} \\
\hline & $n$ & $\bar{x}$ & $s d$ & range & $n$ & $\bar{x}$ & $s d$ & range & \\
\hline Metabolic equivalent & 12 & -3.8 & 1.9 & $-6.7 ;-0.7$ & 13 & -3.0 & 1.6 & $-5.4 ;-0.3$ & 0.323 \\
\hline Maximal oxygen uptake & 12 & -722.8 & 337.9 & $-1120 ;-207$ & 13 & -583.7 & 291.1 & $-1153 ;-99$ & 0.280 \\
\hline Maximal heart rate & 12 & -23.8 & 4.7 & $-29 ;-14$ & 13 & -25.5 & 6.1 & $-40 ;-16$ & 0.422 \\
\hline Max. $\mathrm{VO}_{2}$ at a heart rate & 12 & -2.7 & 1.9 & $-5.5 ; 0.2$ & 13 & -1.6 & 1.9 & $-4.8 ; 2.0$ & 0.155 \\
\hline
\end{tabular}

Table 5. Comparisons between two groups (no bone marrow transplant versus bone marrow transplant) for 4 cardiovascular parameters

\begin{tabular}{|c|c|c|c|c|c|c|c|c|c|}
\hline & \multicolumn{4}{|c|}{$\begin{array}{l}\text { Treatment group } \\
\text { No bone marrow } \\
\text { transplant } \\
N=30\end{array}$} & \multicolumn{4}{|c|}{$\begin{array}{c}\text { Treatment group } \\
\text { Bone marrow } \\
\text { transplant } \\
N=5\end{array}$} & \multirow[t]{2}{*}{$p$} \\
\hline & $n$ & $\bar{x}$ & $s d$ & range & $n$ & $\bar{x}$ & $s d$ & range & \\
\hline Metabolic equivalent & 30 & -3.3 & 1.8 & $-6.7 ;-0.3$ & 5 & -4.5 & 2.9 & $-8.7 ;-1.3$ & 0.174 \\
\hline Maximal oxygen uptake & 30 & -655.6 & 327.3 & $-1302 ;-99$ & 5 & -770.4 & 445.6 & $-1387 ;-318$ & 0.494 \\
\hline Maximal heart rate & 30 & -24.3 & 5.9 & $-40 ;-10$ & 5 & -24.8 & 5.6 & $-31 ;-16$ & 0.860 \\
\hline Max. $\mathrm{VO}_{2}$ at a heart rate & 30 & -2.2 & 2.0 & $-6.2 ; 2.0$ & 5 & -3.2 & 2.3 & $-6.4 ;-0.9$ & 0.325 \\
\hline
\end{tabular}


Table 6. Comparisons between two groups (no steroids versus steroids) for 4 cardiovascular parameters

\begin{tabular}{|c|c|c|c|c|c|c|c|c|c|}
\hline & \multicolumn{4}{|c|}{$\begin{array}{c}\text { Treatment group } \\
\text { No steroids } \\
N=12\end{array}$} & \multicolumn{4}{|c|}{$\begin{array}{c}\text { Treatment group } \\
\text { Steroids } \\
N=23\end{array}$} & \multirow[t]{2}{*}{$p$} \\
\hline & $n$ & $\bar{x}$ & $s d$ & range & $n$ & $\bar{x}$ & $s d$ & range & \\
\hline Metabolic equivalent & 12 & -3.0 & 1.4 & $-5 ;-0.6$ & 23 & -3.8 & 2.3 & $-8.7 ;-0.3$ & 0.295 \\
\hline Maximal oxygen uptake & 12 & -556.8 & 296.5 & $-1153 ;-132$ & 23 & -732.1 & 353.5 & $-1387 ;-99$ & 0.152 \\
\hline Maximal heart rate & 12 & -25.6 & 3.3 & $-33 ;-21$ & 23 & -23.7 & 6.7 & $-40 ;-10$ & 0.282 \\
\hline Max. $\mathrm{VO}_{2}$ at a heart rate & 12 & -1.6 & 1.8 & $-4.8 ; 2.0$ & 23 & -2.7 & 2.2 & $-6.4 ; 1.9$ & 0.167 \\
\hline
\end{tabular}

Table 7. Comparisons between two groups (no central nervous system irradiation versus central system irradiation) for 4 cardiovascular parameters

\begin{tabular}{|l|r|r|r|r|r|r|r|r|r|}
\cline { 2 - 8 } \multicolumn{1}{c|}{} & \multicolumn{3}{c|}{$\begin{array}{c}\text { Treatment group } \\
\text { No central nervous } \\
\text { system irradiation }\end{array}$} & \multicolumn{3}{c|}{$\begin{array}{c}\text { Treatment group } \\
\text { Central nervous } \\
\text { system irradiation }\end{array}$} & $p$ \\
\cline { 2 - 8 } & \multicolumn{1}{c|}{$\bar{x}$} & \multicolumn{1}{c|}{$s d$} & range & $n$ & \multicolumn{1}{c|}{$\bar{x}$} & \multicolumn{1}{c|}{$s d$} & \multicolumn{1}{c|}{ range } & \\
\hline Metabolic equivalent & 23 & -3.4 & 1.9 & $-6.7 ;-0.3$ & 12 & -3.8 & 2.1 & $-8.7 ;-0.7$ & 0.567 \\
Maximal oxygen uptake & 23 & -631.3 & 344.5 & $-1302 ;-99$ & 12 & -750.1 & 335.2 & $-1387 ;-207$ & 0.336 \\
Maximal heart rate & 23 & -24.9 & 6.0 & $-40 ;-10$ & 12 & -23.4 & 5.3 & $-31 ;-14$ & 0.487 \\
Max. $\mathrm{VO}_{2}$ at a heart rate & 23 & -2.0 & 2.2 & $-6.2 ; 2.0$ & 12 & -2.8 & 1.8 & $-6.4 ; 0.2$ & 0.284 \\
\hline
\end{tabular}

There are no differences in the mean values for the chosen cardiovascular parameters between the patients who finished treatment less than 5 years earlier and the patients who finished treatment more than 5 years earlier (Table 4); between the patients who took steroids and those who did not (Table 5); between the patients who underwent irradiation of the central nervous system and those who did not (Table 7); and between the patients who took steroids during treatment and those who did not (Table 6).

Multivariate Normal Model. When performing hypothesis testing separately for the four variables, the fact that these attributes may be correlated was omitted. This may have influenced type I error as well as the obtained results. If correlation is present and significant, two methods can be used. The first one is to apply the principal component analysis, especially if the number of variables in a data set is large and dimensionality reduc- 
tion is reasonable and done simultaneously. The second approach is to build a multivariate normal model that allows to correct standard errors and perform inference. In this paragraph, the second method is described to present the results of comparison between the mean values for the treated and the healthy children.

To confirm the statistically significant results for the differences between the treatment and the control group in the whole dataset, the multivariate normal model was built with the use of the SAS system. Four cardiovascular variables were included in the model.

The basic obtained model was as follows:

$$
\begin{aligned}
Y_{i j}= & -1.227-1.084 \cdot \text { group }_{i}-1.3 \cdot d_{\_} \text {met }_{j}-520.26 \cdot d_{-} \text {peakvo }_{j}- \\
& 26.746 \cdot d_{-} \text {maxhr }_{j}+0.111 \cdot \text { group }_{i} \cdot d_{-} \text {mets }_{j}-149.43 \cdot \text { group }_{i} \cdot d_{-} \text {peakvo2 }_{j} \\
& +4.686 \cdot \text { group }_{i} \cdot d_{\_} \text {maxhr }_{j},
\end{aligned}
$$

where: group is a dummy variable for the treatment group; d_mets, d_peakvo2, d_maxhr are dummy variables for respectively: metabolic equivalent (mets), maximal oxygen uptake (peakvo2), and maximal heart rate (maxhr). As a baseline, the control group and maximal $\mathrm{VO}_{2}$ at a heart rate (maxvo2hr) were chosen.

Finally, the multivariate model was formed:

$$
\left(\begin{array}{c}
\text { mets }_{i} \\
\text { peakvo2 }_{i} \\
\text { maxhr }_{i} \\
\text { maxvo2hr }_{i}
\end{array}\right)=N\left(\left(\begin{array}{c}
-2.527-0.973 \cdot \text { group }_{i} \\
-521.487-150.514 \cdot \text { group }_{i} \\
-27.973+3.6015 \cdot \text { group }_{i} \\
-1.227-1.0844 \cdot \text { group }_{i}
\end{array}\right), \Sigma\right) \text {, }
$$

where $\Sigma$ is the covariance matrix with the following variances $3.49,145031$, $33.1592,4.7944$. The unstructured type of the working correlation matrix was chosen to obtain the results and the corrected standard errors.

The estimated mean values for the four variables and the two groups are presented in Table 8 . The estimators assess real marginal mean values, through a precise comparison of the estimates in Table 1.

The next step was to test if there were any differences between mean values for the variables. Looking at the multivariate normal model, it should be sufficient to test if the coefficients of the dummy variable group (treatment group) for the four equations are different than zero. Below, $\beta_{1}, \beta_{2}, \beta_{3}$ and $\beta_{4}$ are used to denote these coefficients. If null hypotheses are rejected, it is equivalent to the statistically significant difference between the mean values between the treatment group and the control groups. For the three parameters $\beta_{1}, \beta_{3}$ and $\beta_{4}$, the hypotheses that each equals zero were rejected 
Magdalena Topczewska et al.

Table 8. Least square means

\begin{tabular}{|cl|r|r|r|r|c|}
\hline \multirow{2}{*}{ group } & variable & estimate & $\begin{array}{c}\text { standard } \\
\text { error }\end{array}$ & df & t statistic & $\mathrm{p}$ \\
\hline \multirow{6}{*}{ treatment } & mets & -3.50 & 0.32 & 70 & -11.07 & $<.0001$ \\
& peakvo2 & -672.00 & 64.37 & 70 & -10.44 & $<.0001$ \\
& maxhr & -24.37 & 0.97 & 70 & -25.04 & $<.0001$ \\
& maxvo2hr & -2.31 & 0.37 & 70 & -6.25 & $<.0001$ \\
\hline \multirow{6}{*}{ control } & mets & -2.53 & 0.31 & 70 & -8.22 & $<.0001$ \\
& peakvo2 & -521.49 & 62.61 & 70 & -8.33 & $<.0001$ \\
& maxhr & -27.97 & 0.95 & 70 & -29.55 & $<.0001$ \\
& maxvo2hr & -1.23 & 0.36 & 70 & -3.41 & 0.0011 \\
\hline
\end{tabular}

( $p=0.03 ; p=0.01 ; p=0.04$, respectively); thus, this may lead to the conclusion that there are differences in metabolic equivalent, maximal heart rate and maximal $\mathrm{VO}_{2}$ at a heart rate between the control group and the treatment group, which confirms the general results described in the previous paragraph.

Classification. The last of the approaches presented in this paper did not constitute an attempt to assess the differences in parameters, but rather to build a classification model to describe the relationship between the group attribute and cardio-respiratory variables. The aim was to check its classification usefulness and properties, and then to separate objects belonging to one of the two groups: treatment and control. Logistic regression was applied as the method of classification with robust estimates of variance.

The model containing the original variables is not presented due to the fact that a strong correlation was noticed among the variables (Table 9), which has an impact on the estimated parameters. To remove the correlation, principal component analysis was applied. The resultant data is at different scales depending on the variable, which the PCA is sensitive to. For this reason, PCA based on the correlation matrix, rather than on the covariance matrix, is calculated.

Results describing the contribution of eigenvalues for the principal components in total sample variance division and factor loadings for the four principal components are presented in Table 10. The first principal component explains $70 \%$ of the total sample variance. The first two principal components, collectively, explain $96 \%$ of the total sample variance. The first three principal components explain 99\%, while the first four $-100 \%$ of the total sample variance. In conclusion, sample variation is summarized 
Statistical Methods in the Evaluation of Cardio-Respiratory Parameters...

Table 9. Correlation matrix of the selected variables of the cardio-respiratory system

\begin{tabular}{|l|l|l|l|c|}
\hline & mets & peakVO2 & maxhr & maxVO2hr \\
\hline mets & 1.000 & & & \\
peakVO2 & $0.857^{*}$ & 1.000 & & \\
maxhr & 0.172 & 0.071 & 1.000 & \\
maxVO2hr & $0.918^{*}$ & $0.925^{*}$ & -0.032 & 1.000 \\
\hline
\end{tabular}

Table 10. Contribution of eigenvalues for principal components in total sample variance division and factor loadings for the four principal components

\begin{tabular}{|c|c|c|c|c|c|}
\hline & & $\begin{array}{c}\text { Principal } \\
\text { component } \\
\text { no } 1\end{array}$ & $\begin{array}{c}\text { Principal } \\
\text { component } \\
\text { no } 2\end{array}$ & $\begin{array}{c}\text { Principal } \\
\text { component } \\
\text { no } 3\end{array}$ & $\begin{array}{c}\text { Principal } \\
\text { component } \\
\text { no } 4\end{array}$ \\
\hline & Eigenvalue & 2.81 & 1.01 & 0.14 & 0.04 \\
\hline Cumulat & e eigenvalue & 0.70 & 0.96 & 0.99 & 1.00 \\
\hline \multirow{4}{*}{ factor loadings } & mets & 0.573 & 0.074 & -0.679 & -0.452 \\
\hline & peakVO2 & 0.572 & -0.041 & 0.728 & -0.375 \\
\hline & $\operatorname{maxh} r$ & 0.067 & 0.986 & 0.073 & 0.136 \\
\hline & $\max V O 2 h r$ & 0.583 & -0.145 & -0.056 & 0.798 \\
\hline
\end{tabular}

very well by the first two principal components. A Kaiser-Meyer-Olkin Measure of Sampling Adequacy of 0.597 means that principal component analysis can be used in the situation in question. A reduction of data to 2 principal components is reasonable, particularly in the context of a correlation between the attributes.

On the basis of Kaiser's rule, only two principal components should be retained in further analysis, i.e. only for the two components the corresponding eigenvalues exceeded 1, i.e. 2.81 and 1.01 (Table 10). When analyzing the factor loadings presented in Table 10, absolute values larger than 0.3 were considered. The first principal component describes a linear relationship between metabolic equivalent (mets), maximal oxygen uptake (peakVO2), and maximal $\mathrm{VO}_{2}$ at a heart rate (maxVO2hr), while the second principal component is influenced only by maximal heart rate (maxhr).

Then, principal components were applied to build a classification model using the logistic regression approach. The obtained coefficient estimates and robust standard errors, as well as the odds ratios and the corresponding 
Magdalena Topczewska et al.

Table 11. Results of the logistic regression approach

\begin{tabular}{|c|cc|ccc|}
\hline & Coef. & Std.Err $_{\text {Coef }}$ & OR & Std.Err $_{\text {OR }}$ & 95\%CI for OR \\
\hline Principal comp. no 1 & -0.311 & 0.158 & 0.733 & 0.116 & $0.537 ; 0.998$ \\
Principal comp. no 2 & 0.758 & 0.279 & 2.133 & 0.596 & $1.234 ; 3.690$ \\
Const. & -0.088 & 0.260 & - & - & - \\
\hline
\end{tabular}

robust standard errors, are presented in Table 11, for the whole group of patients. The use of the two variables in the model is reasonable, as the model is statistically significant $(p=0.003)$.

On the basis of the Hosmer-Lemeshow goodness-of-fit test, the model cannot be rejected (Hosmer-Lemeshow $\chi^{2}=12.63 ; d f=8 ; p=0.125$ ). Statistical significance was achieved for both components: the first principal component $(p=0.049)$ and the second principal component $(p=0.007)$. When using the PCA approach, simple interpretability of parameters was lost; however, general conclusions can still be formulated. The coefficient estimate $(-0.311)$, and hence the odds ratio associated with the first principal component (0.733), indicate that in the case of an increase of the value in the set consisting of metabolic equivalent, maximal oxygen uptake, and maximal $\mathrm{VO}_{2}$ at a heart rate, the odds decrease. Thus, deviation from the norm for the three original variables is larger for the treatment group compared to the control group. For the second principal component, an opposite result (coef. estimate $=0.758$, OR $=2.133$ ) can be observed, thus the deviation from the norm is larger for the control group. The results are similar to those obtained for the two different approaches presented in the previous paragraphs.

Detailed classification characteristics for the treated and the healthy patients are shown in Table 12 (model for the whole group: $\mathrm{M}_{\text {Overall }}$ column). When trying to separate the treated children from those healthy on the basis of cumulated information on the parameters of their cardiovascular systems, as low an accuracy as $62.5 \%$ was obtained with an AUC of 0.715 . The ROC curve is shown in Figure 3a, while the sensitivity and specificity versus probability cutoff relation is presented in Figure 3b. After applying the crossvalidation technique for the classification of these values, they were $63.89 \%$ and 0.676, respectively. All the detailed classification characteristics such as sensitivity, specificity, and positive and negative predictive values were at a level of $62-66 \%$, which is not high.

Two additional models were built to check trends in differences between variables, separately in each of the gender subgroups (Table 13). On the basis 
Statistical Methods in the Evaluation of Cardio-Respiratory Parameters...

Table 12. Detailed classification characteristics

\begin{tabular}{|l|l|l|l|}
\hline & $\mathrm{M}_{\text {Overall }}$ & $\mathrm{M}_{\text {Female }}$ & $\mathrm{M}_{\text {Male }}$ \\
\hline Accuracy & $62.50(63.89)$ & $78.95(76.32)$ & $58.82(58.82)$ \\
Sensitivity & $62.86(65.7)$ & $83.33(77.8)$ & $58.82(58.8)$ \\
Specificity & $62.16(62.2)$ & $75.00(75.0)$ & $58.82(58.8)$ \\
Positive predictive value & $61.11(62.2)$ & $75.00(73.7)$ & $58.82(58.8)$ \\
Negative predictive value & $63.89(65.7)$ & $83.33(78.9)$ & $58.82(58.8)$ \\
ROC Area & $0.715(0.676)$ & $0.833(0.744)$ & $0.668(0.561)$ \\
\hline
\end{tabular}

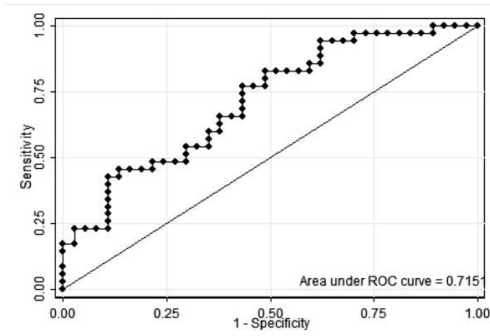

a) ROC curve plot

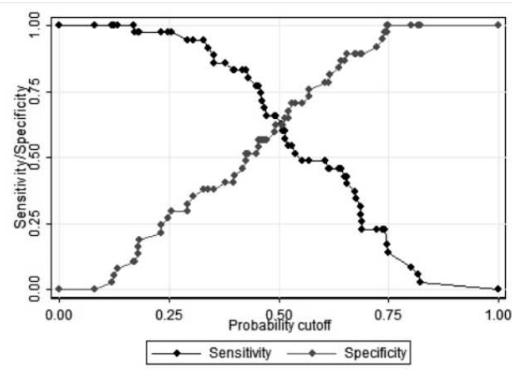

b) Sensitivity and specificity versus probability cutoff

Figure 3. Classification quality assessment

Table 13. Results of the logistic regression approach for the female and the male subgroups

\begin{tabular}{|c|c|rc|ccc|}
\hline & & Coef. & Std. & & Orr & \\
foef & OR & Std.Err $_{\text {OR }}$ & 95\%CI for OR \\
\hline \multirow{2}{*}{ female } & Principal comp. no 1 & -0.863 & 0.435 & 0.422 & 0.184 & $0.179 ; 0.990$ \\
& Principal comp. no 2 & 0.813 & 0.381 & 2.254 & 0.858 & $1.069 ; 4.753$ \\
& Const. & 0.342 & 0.493 & - & - & - \\
\hline \multirow{3}{*}{ male } & Principal comp. no 1 & -0.126 & 0.212 & 0.881 & 0.187 & $0.582 ; 1.335$ \\
& Principal comp. no 2 & 0.809 & 0.476 & 2.247 & 1.069 & $0.884 ; 5.711$ \\
& Const. & -0.269 & 0.422 & - & - & - \\
\hline
\end{tabular}

of the Hosmer-Lemeshow goodness-of-fit test, the model for the female subgroup cannot be rejected $\left(\chi^{2}=10.39 ; d f=8 ; p=0.238\right)$. The use of both variables in the model is reasonable and the model is statistically significant $(p=0.043)$. Statistical significance was achieved for both components: the first principal component $(p=0.047)$ and the second principal component 
$(p=0.033)$. Deviations from the norm for the three original variables are larger in the treatment group compared to the control group than in the whole group, thus the survivor females' fitness is lower level. On the other hand, in the male subgroup the model is not significant $(p=0.225)$, with the following values for both parameters: $p=0.551$ for the odds ratio of the first principal component and $p=0.089$ for the odds ratio of the second principal component. No statistically significant differences between the males belonging to the treatment group and those belonging to the control group as far as deviations from the norm are concerned could be concluded.

All classification statistics for the female subgroup are much higher compared to the whole group of patients (Table 12, column $\mathrm{M}_{\mathrm{Female}}$ ). The classification accuracy for the female subgroup only is $78.95 \%$ (with the usage of cross-validation technique $76.32 \%$ ); sensitivity: $83.33 \%$ (77.8\%); specificity: $75.0 \%$ (75.0\%); positive predictive value: $75.0 \%$ (73.7\%); negative predictive value: $83.33 \%(78.9 \%)$. The area under the curve equals $0.833(0.744)$ (Figure 4a, 4b).

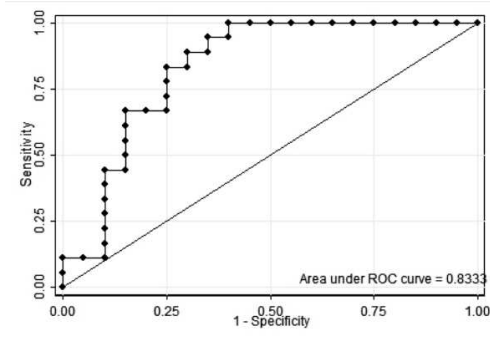

a) ROC curve for the female subgroup

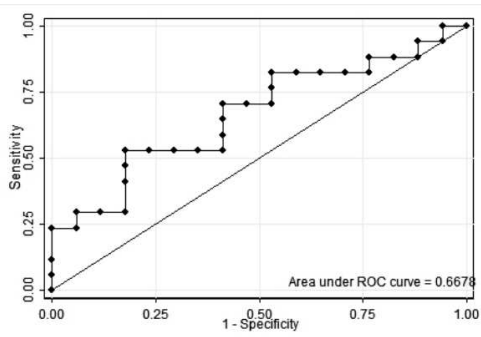

c) ROC curve for the male subgroup

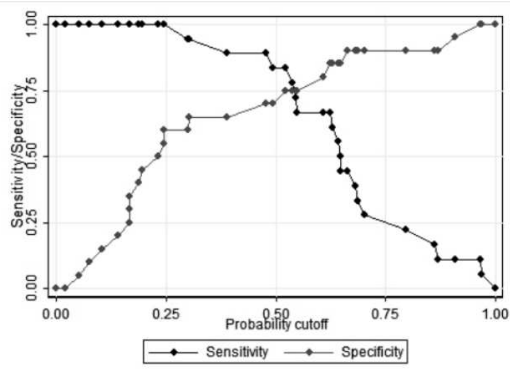

b) Sensitivity and specificity versus probability cutoff for the female subgroup

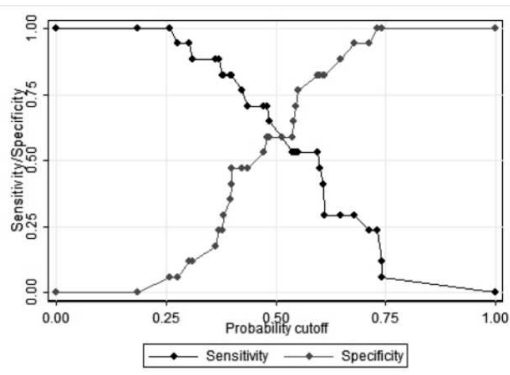

d) Sensitivity and specificity versus probability cutoff for the male subgroup

Figure 4. Classification quality assessment

The results indicate that cumulative information about the selected cardiorespiratory system parameters differ between the treated and the healthy 
females. On the other hand, the detailed classification statistics in the case of the male subgroups confirm that there is no evidence of a difference in deviations from the norm between the treated and the healthy males in the context of fitness level (Table 12, column $\mathrm{M}_{\text {Male }}$; Figure 4c, 4d).

\section{Conclusions}

Reduced parameters of the cardiorespiratory system in childhood may have a tremendous health impact and lead to irreversible consequences in adulthood. Three different approaches, from the field of statistics and machine learning, were applied to check the results of four cardiorespiratory system parameters and the results were compared with those obtained for healthy children.

The conclusions are similar in the case of all the methods. Disturbances in the selected parameters of the cardiorespiratory system among the convalescent confirm worse exercise tolerance compared to their peers. These differences are particularly noticeable in the female subgroup.

Long-term observation would confirm our results. However, knowing the trends in data and being aware of the consequences would enable the introduction of targeted prevention programs. Disease prevention becomes one of the main objectives after treatment and relates particularly to young survivors.

\section{R E F E R E N C E S}

Bacha, F., \& Gidding, S. S. (2016). Cardiac abnormalities in youth with obesity and type 2 diabetes. Current Diabetes Reports, 16(7): 62. doi: 10.1007/s11892016-0750-6

Beck, A. R. (2016). Psychosocial aspects of obesity. NASN School Nurse, 31(1), 23-27. doi: 10.1177/1942602X15619756

Cote, A. T., Harris, K. C., Panagiotopoulos, C., Sandor, G. G., \& Devlin, A. M. (2013). Childhood obesity and cardiovascular dysfunction. Journal of the American College of Cardiology, 62(15), 1309-1319. doi: 10.1016/j.jacc.2013. 07.042

Dietz, W. H. (1998). Health Consequences of Obesity in Youth: Childhood Predictors of Adult Disease. Pediatrics, 101 (Suppl. 2), 518-525.

Jetté, M., Sidney, K., \& Blümchen, M. (1990). Metabolic Equivalents (METS) in Exercise Testing, Exercise Prescription, and Evaluation of Functional Capacity. Clinical Cardiology, 13(8), 555-565. doi: 10.1002/clc.4960130809 
Lauer, R. M., \& Clark, W. R. (1989). Childhood Risk Factors for High Adult Blood Pressure: The Muscatine Study. Pediatrics, 84(4), 633-641.

Leiba, A., Kark, J. D., Afek, A., Levi, Z., Barchana, M., Tzur, D., Derazne, E., et al. (2012). Overweight in Adolescence is Related to Increased Risk of Future Urothelial Cancer. Obesity, 20(12), 2445-2450. doi: 10.1038/oby.2012.83

Lloyd, L. J., Langley-Evans, S. C., \& McMullen, S. (2012). Childhood obesity and risk of the adult metabolic syndrome: a systematic review. International Journal of Obesity (Lond), 36, 1-11. doi: 10.1038/ijo.2011.186

McCrindle, B. W. (2015). Cardiovascular Consequences of Childhood Obesity. Canadian Journal of Cardiology, 31(2), 124-130. doi: 10.1016/j.cjca.2014.08. 017

Mohanan, S., Tapp, H., McWilliams, A., \& Dulin, M. (2014). Obesity and asthma: Pathophysiology and implications for diagnosis and management in primary care. Experimental Biology and Medicine (Maywood), 239(11), 1531-1540. doi: $10.1177 / 1535370214525302$

Morrison, K. M., Shin, S., Tarnopolsky, M., \& Taylor, V. H. (2015). Association of depression and health related quality of life with body composition in children and youth with obesity. Journal of Affective Disorders, 172, 18-23. doi: $10.1016 /$ j.jad.2014.09.014

Narang, I., \& Mathew, J. L. (2012). Childhood Obesity and Obstructive Sleep Apnea. Journal of Nutrition and Metabolism, 2012: 134202. doi: 10.1155/2012/ 134202

Pollock, N. K. (2015). Childhood obesity, bone development, and cardiometabolic risk factors. Molecular and Celularl Endocrinology, 410, 52-63. doi: 10.1016/ j.mce.2015.03.016

Topczewska, M., Sawicka-Żukowska, M., \& Krawczuk-Rybak, M. (2014). Statistical and Multidimensional Body Composition Parameter Analysis in Young Childhood Cancer Survivors. Studies in Logic, Grammar and Rhetoric. Logical, Statistical and Computer Methods in Medicine, 39(52), 25-42. doi: $10.2478 /$ slgr-2014-0051 